\title{
Shear turbulence on a sparse spectral grid
}

\author{
F. De Lillo and Bruno Eckhardt \\ Fachbereich Physik, Philipps-Universität Marburg, D-35032 Marburg, Germany
}

(Received 5 February 2007; published 3 July 2007)

\begin{abstract}
We simulate turbulence in a plane Couette geometry by a spectral method intermediate between full resolution and the complete elimination of small modes common in large eddy simulations. The wave number grid is sparse in spanwise and downstream direction, with a total number of modes proportional to $\mathrm{Re}^{3 / 4} \ln \mathrm{Re}$. At a Reynolds number of 2000 we could suppress more than $80 \%$ of the modes and still obtain a fairly accurate resolution of the boundary layer structures and friction factors. For a wide range of resolutions, the mean velocities are described by logarithmic profiles, with von Karman constant near a value of 0.4.
\end{abstract}

DOI: 10.1103/PhysRevE.76.016301

PACS number(s): 47.27.nd, 47.27.er, 47.27.eb, 47.27.E-

\section{INTRODUCTION}

Direct numerical simulations (DNS) of turbulent flows provide access to the velocity fields at all times and locations, at the price of an often enormous computational effort, already at moderate Reynolds numbers [1]. This effort is further increased by the long time averages needed for the study of highly non-Gaussian quantities, such as small scale velocity increments. For many applications, on the other hand, one can expect that the properties of the flow are captured by the energy carrying, large scale modes and that, therefore, a simulation using a suitable model for the small scales may reproduce the dominant features. A prominent class of models with this philosophy are large eddy simulations (LES), where different models are distinguished by the different approaches to the replacement of the small scale structures [2-6]. The quality of such an approach evidently rests on the accuracy with which the small scales are represented, and it becomes a delicate issue in situations where they are significant [2]: In the frequently arising case of wallbounded turbulence, the large scale turbulence in the bulk is (re)generated by small scale processes that take place in the high stress near-wall region. Application of LES to wall bounded flows therefore requires an accurate modeling of the whole viscous layer $[2,6]$.

Intermediate between the direct numerical simulations with full spectral resolution and large eddy simulations where the small scales are completely eliminated are simulations on sparse grids or with a reduced set of wave numbers [7-12]. These models are inspired by the turbulent cascade and are hence most easily described in wave number space. Consider the case of a turbulent flow with velocity fields expanded in Fourier series $u_{i}(\mathbf{x}, t)$ $=\sum_{\mathbf{k}} u_{i}(\mathbf{k}, t) \exp (i \mathbf{x} \cdot \mathbf{k})$, obeying the Navier-Stokes equation

$$
\frac{\partial u_{i}(\mathbf{k})}{\partial t}=-i M_{i j l}(\mathbf{k}) \sum_{\mathbf{p}} u_{j}(\mathbf{p}) u_{l}(\mathbf{k}-\mathbf{p})-\nu \mathbf{k}^{2} u_{i}(\mathbf{k}),
$$

where $M_{i j l}(\mathbf{k})=\left[k_{j} P_{i l}(\mathbf{k})+k_{l} P_{i j}(\mathbf{k})\right] / 2$ with $P_{i j}(\mathbf{k})$ the projector normal to the wave vector $\mathbf{k}$, in order to assure incompressibility. Equation (1) shows that the modes in the nonlinear term interact in pairs to contribute to a third mode whose wave number is the sum of their individual wave numbers. This property of the interactions can be preserved while reducing the number of modes used to represent the fields. In particular, it is possible to model arbitrarily small scales by keeping a few high wave number modes together with threads that connect them to low wave number modes. A simple version of such a grid can be built as follows: start with a core group of wave numbers, for instance by selecting all the wave vectors $\mathbf{k}$ of modulus smaller than $k_{c}$; then add wave vectors $\mathbf{k}$ to the grid if there is a $\mathbf{K}$ in the core group such that $\mathbf{k}=\lambda^{n} \mathbf{K}$ for some integer $n=1,2, \ldots$. The scaling $\lambda$ is prescribed and fixed, and most often (also here) taken to be $\lambda=2$. The mode reduction is completed by defining the equations of motion to be the restriction of Eq. (1) to the reduced set. With this definition, the structure of the mode-to-mode interaction is preserved, the nonlinear terms are energy conserving and all scales, from the largest to the smallest, have some dynamical degrees of freedom. These models were studied primarily in the context of homogeneous isotropic turbulence $[8,12]$, in Rayleigh-Benard flows $[13,14]$, in viscoelastic turbulence [15] and in magnetohydrodynamic turbulence [7]. Slight variations in the choice of the base set of modes can be found in the literature $[7,8]$.

The computational effort that goes with these models is much reduced compared to the full DNS. Estimates for homogeneous turbulence based on Kolmogorov's theory suggest that in order to resolve all scales down to the dissipative scale $\eta$ in a box of side length $L$, wave numbers up to $k_{\eta}$ $\sim L / \eta \sim \mathrm{Re}^{3 / 4}$ are required, so that the total number of modes increases like $\mathrm{Re}^{9 / 4}$ [16]. For the sparse grids, the upscaling from the maximal wave number $k_{c}$ in the core group to the maximal wave number $k_{\eta}$ needed is achieved by the algebraic upscaling with $\lambda^{n}$, so that the total number of wave vectors increases only as $\ln k_{\max } / k_{\eta} \sim \ln \mathrm{Re}$. The price to be paid is that the reduction of wave numbers cuts some of the couplings between large and small scales and hence partially suppresses intermittency [12]. Also, the Batchelor constant in the structure function increases [8]. Nevertheless, mode reduction models have been useful in studying relative changes, such as variations of exponents [10].

Our aim here is to introduce such models for the case of wall bounded flows and to study the effects of modal reduction on friction, boundary layer profiles and fluctuations. If these quantities are correctly reproduced, mode reduction could provide a way to attain Reynolds numbers several orders of magnitude above the ones that can be handled currently. For our study, we chose the simplest non-isotropic geometry of plane Couette flow, where two parallel walls 


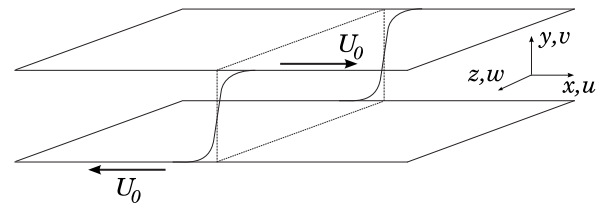

FIG. 1. Schematic representation of the plane-Couette geometry. The coordinate axes $x, y$, and $z$ point in the downstream, normal and spanwise direction. The associated velocity components are labeled $u, v$, and $w$. The velocity of the top and bottom plates is $+U_{0}$ and $-U_{0}$, respectively, and the gap width is $2 h$. No-slip boundary conditions are assumed on the walls, and the down-stream and spanwise directions are assumed to be periodic.

moving in opposite direction drive a flow by the friction at the walls (Fig. 1). For this geometry relatively straightforward spectral algorithms $[17,18]$ are available. In the downstream and spanwise directions periodic boundary conditions can be adopted, and a Fourier decomposition can be used. For the wall-normal direction we chose Chebyshev modes. In this way all required transformations can be performed by means of efficient fast Fourier transform (FFT) routines. Moreover, the spacing of the Gauss-Lobatto points on which the fields are represented decreases near the walls, thus assuring us of a high and accurate resolution of the large velocity gradients and the small scale dynamics in the nearwall region that is so fundamental for the regeneration of turbulence [19-22]. The key difference to the homogeneous isotropic case is that spectral reduction is applied in planes parallel to the wall only, and that the full resolution is kept in the wall normal direction. Since in the latter the classical scaling must be applied, a total number of modes proportional to $\mathrm{Re}^{3 / 4} \ln \mathrm{Re}$ should be necessary for a run at fixed Re.

In the next section we summarize the numerical procedure. Section III contains the results on mean profiles and the friction factor. Near wall structures are discusssed in Sec. IV, and spectra and velocity structure functions in Sec. V. We close with some remarks and an outlook on future developments in Sec. VI.

\section{THE NUMERICAL PROCEDURE}

The Navier-Stokes equations were solved through a Chebyshev- $\tau$ scheme [17]. Periodic boundary conditions were imposed in the streamwise and spanwise directions, and no-slip conditions at the walls $\left[\mathbf{u}(y= \pm 1)=0,\left.\partial_{y} v\right|_{y= \pm 1}=0\right]$. Fields are decomposed in Fourier series along the periodic directions and in Chebyshev series in the wall-normal one. The equations for the wall-normal components of velocity $v$ and vorticity $\omega_{y}$ were solved,

$$
\left\{\begin{array}{l}
\frac{\partial v}{\partial t}+\mathbf{u} \cdot \nabla v=-\nabla p+\nu \Delta v \\
\frac{\partial \omega_{y}}{\partial t}+[\boldsymbol{\nabla} \times(\mathbf{u} \cdot \nabla \mathbf{u})]_{y}=\nu \Delta \omega_{y}
\end{array}\right.
$$

and the remaining components of velocity were computed by making use of the incompressibility condition $\boldsymbol{\nabla} \cdot \mathbf{u}=0$.

The code is fully dealiased and was tested by analysis of the decay of small perturbations of the laminar profile (where correct eigenvalues are measured for the eigenmodes of the linearized operator [23]), and by comparison with published numerical and experimental results.

After performing a reference run with full numerical resolution, we repeated the evolution of the same initial field on reduced sets of modes. In the current simulations, a modified version of the code where the unwanted modes are put to zero before and after the computation of the nonlinear term was used. Clearly, in this implementation nothing is gained computationally from mode reduction, our aim here being only to test its effects on physical observables. On the other hand, a code built in this way is extremely flexible in the definition of the computational grid. The final, fully optimized implementation will be indeed very different from this, and will depend on the specific choice of the reduced set of modes. An example of such a code can be found in Ref. [7], where FFT's of reduced size are used to compute the convolutions. In the simulations we present here the grid was built from a core group containing all modes with wave vectors $\left(k_{x}, k_{z}\right)$ such that $\left|k_{x}\right|,\left|k_{z}\right|<n_{c}$ and their upscaled partners $\mathbf{k}_{i, n}=2^{n} \mathbf{K}_{i}$, with $K_{i}$ a wave vector of the core group. In what follows we present the results of simulations performed with constant bulk Reynolds number for different values of $n_{c}$.

Coordinates for our simulation are $x$ in the streamwise, $y$ in the wall normal, and $z$ in the spanwise direction. The corresponding velocity components are $u, v$, and $w$ (see Fig. $1)$. In what follows the notation $\langle\cdots\rangle_{y}$ denotes an average

TABLE I. Parameters for the simulations on the sparse and full grid. $n_{c}$ parametrizes the size of the core group, $N_{k}$ is the number of active $2 D$ wave numbers kept in the grid, $\tau$ is the wall friction, $u_{*}$ the friction velocity, and $\mathrm{Re}_{*}$ the friction Reynolds number. The completely resolved DNS has $256 \times 128$ Fourier modes in the plane and is labeled FR. The actual number of active modes is smaller because of the truncation performed for dealiasing. Due to the reality condition on Fourier modes, only half of the modes really enter the computation. In all cases the wall speed $U_{0}=1$, and the kinematic viscosity $\nu=5 \times 10^{-4}$. The last column shows the linestyle used in all plots for reference.

\begin{tabular}{lcccccc}
\hline \hline & $n_{c}$ & $N_{k}$ & $10^{3} \tau$ & $10^{2} u^{*}$ & $\mathrm{Re}_{*}$ & \\
\hline$a$ & 8 & 822 & 4.11 & 6.41 & 128 & $\ldots$ \\
$b$ & 16 & 2338 & 4.03 & 6.35 & 127 & - \\
$c$ & 24 & 4262 & 3.78 & 6.15 & 123 & $-\cdots$ \\
$d$ & 32 & 6142 & 3.59 & 5.99 & 120 & $-\cdot-$ \\
FR & 14362 & 3.59 & 5.99 & 120 & - \\
\hline \hline
\end{tabular}






FIG. 2. Streamwise velocity profiles from the simulation at full resolution (full line) and from several sparse grid runs (see Table I for the relative line styles). While the main shape of the profiles is preserved, the inset shows that the average vertical gradients near the wall increase monotonically with the size of the core group.

over time and streamwise and spanwise coordinates in planes at a fixed vertical coordinate $y$ (the label may be dropped when the average to be taken is unambiguous). The distance from the upper and lower wall is given by $Y=1-y$ and $Y$ $=1+y$, respectively. A reference simulation at full resolution was performed at a Reynolds number $\operatorname{Re}=U_{0} h / \nu=2000$, based on the wall speed $U_{0}$, the half height $h$ of the channel, and the kinematic viscosity $\nu$. The computational grid had a resolution of $256 \times 65 \times 128$ points $\left(N_{x} \times N_{y} \times N_{z}\right)$. The half height of the channel was $h=1$, while the streamwise and spanwise sizes were $L_{x}=4 \pi$ and $L_{z}=2 \pi$ respectively. The friction velocity was determined to be $u_{*}=\sqrt{\tau} \sim 0.06$ (where $\tau=\left.\nu \partial_{y}\langle u\rangle\right|_{y= \pm 1}$ is the average shear stress at the wall), and the friction Reynolds number $\operatorname{Re}_{*}=u * h / \nu=120$. These values are about $10 \%$ above the ones interpolated for the same Re from the experimental data of Ref. [24], but there is perfect agree-


FIG. 3. Running time average of the friction factor $J$, at the wall (a) and at the center line (b), for full resolution and for the reduced simulation at $n_{c}=16$ and 24 , showing the relative statistical errors. Even after long averaging times some residual fluctuations remain (see Table I for the relative line styles).
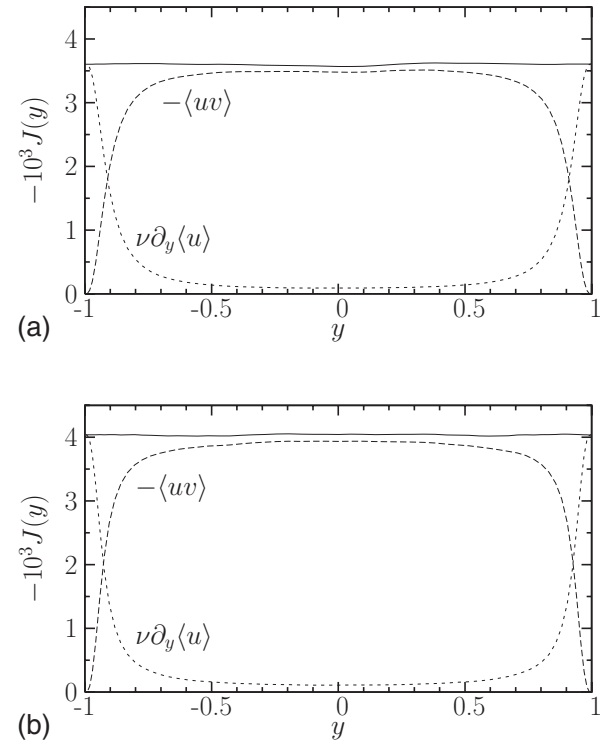

FIG. 4. The friction factor $J$ plotted as a function of the wall normal coordinate (continuous line), for the reference run (a) and for the sparse grid with $n_{c}=16$ (b). The separate contributions from the wall and Reynolds stresses are also plotted, with the latter dominant in the center line.

ment between our $\mathrm{Re}$ vs $\mathrm{Re}_{*}$ curve (obtained from several test runs) and the DNS results of Refs. [25]. For the sparse grid simulations we kept the geometry, the viscosity and the wall speed unchanged, and varied the core group parameter $n_{c}$ only.

\section{RESULTS OF THE SIMULATIONS}

The main parameters of the runs are summarized in Table I. The overall shapes of the velocity profiles are similar to the one from the full simulations (Fig. 2). The data and the inset of Fig. 2 show that mode reduction gives rise to larger wall gradients. However, the run with $n_{c}=16$ shows that for a reduction to only $16 \%$ of the modes the increase in $R *$ is less than $6 \%$. A larger wall stress is the mark of an increased momentum transport. In a plane channel, the average momentum transfer in the wall normal direction is given by $J(y)=\langle u v\rangle_{y}-\nu \partial_{y}\langle u\rangle$. In a statistically stationary Couette flow this quantity is independent of the height $y$. As a consequence of boundary conditions, $\langle u v\rangle_{y= \pm 1}=0$ and $J(y)=J(0)$ $=-\tau$. Figure 3 shows the running time average of $J$ near the wall and at the center line. The first value corresponds exactly to the wall stress, while the second is dominated by the Reynolds stress. The splitting of the current $J$ into the two contributions is shown in Fig. 4 as a function of the wall normal coordinate.

In Fig. 5 the streamwise velocity profiles are shown in the usual semi-logarithmic representation. The velocity and the wall-normal coordinate are expressed in wall units as $u^{+}$ $=u / u^{*}$ and $y^{+}=Y u^{*} / \nu$, where $u^{*}$ is the actual friction velocity of each run. When compared to the logarithmic profile $\left\langle u^{+}\right\rangle$ $=(1 / \kappa) \ln \left(y^{+}\right)+C_{l}[26-28]$ the simulation at full resolution gives $\kappa=0.4$ (the von Karman constant) and $C_{l}=4.7$. The 
simulations for $n_{c} \geqslant 16$ are consistent with a logarithmic law. Further reductions, as for $n_{c}=8$, show a wavy modulation in the profile. While the logarithmic shape is suggested by rather simple and general dimensional [28] and symmetry arguments [29-31], its preservation is not trivial, and indeed the log-law is not observed at the largest reductions. Furthermore, it is remarkable that the value of the von Karman constant measured in the sparse-grid runs agrees with the accepted value, when a sufficient (but still much smaller than for full resolution) number of modes is considered. For the Reynolds number considered here, sufficient means as few as $16 \%$ of all modes, for $n_{c}=16$.

The shift in the logarithmic region is easily seen to be a consequence of the variation in wall shear stress. Indeed, the coefficient can be estimated from the parameters of the simulations as $C_{l}=U_{0} / u^{*}-(1 / \kappa) \ln \left(h u^{*} / \nu\right)$, where the first term is clearly dominant and results in $C_{l} \sim 1 / \sqrt{\tau}$. Substitution of the values of Table I gives good agreement with the fitted values. Another indication of the effects of the mode reduction on near wall structure is given in Fig. 6, where the profiles of the root mean square (rms) values of the streamwise velocity fluctuations are shown. The maximum of $u_{\mathrm{rms}}$ at full resolution is around $y_{+}=14$, consistent with what was found in Refs. [24,32]. While this peak is in the same position for the higher resolutions $n_{c}=32$ and $n_{c}=24$, it moves closer to the wall for lower resolutions.
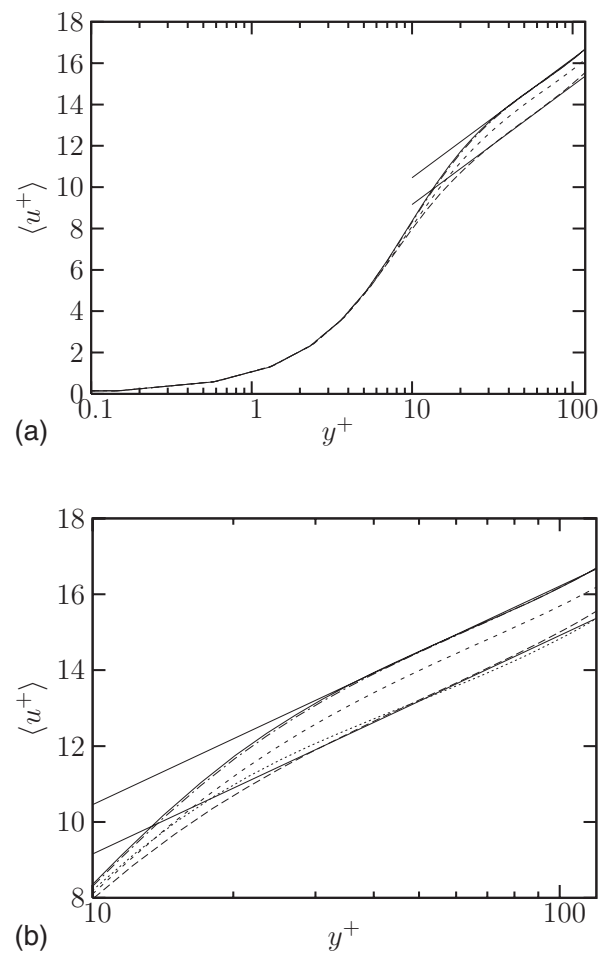

FIG. 5. The streamwise velocity profiles in wall units [magnified around the log region in plot (b), line styles are given in Table I]. Each profile has been normalised by the respective friction velocity. The slope expected for a von Karman constant $\kappa=0.4$ in front of the logarithmic part is shown by the two lines with constants $C_{l}=3.5$ and $C_{l}=4.7$. The latter value is found for the simulation at full resolution. The shift is due to the different values of the wall shear stress (see text for further discussion).

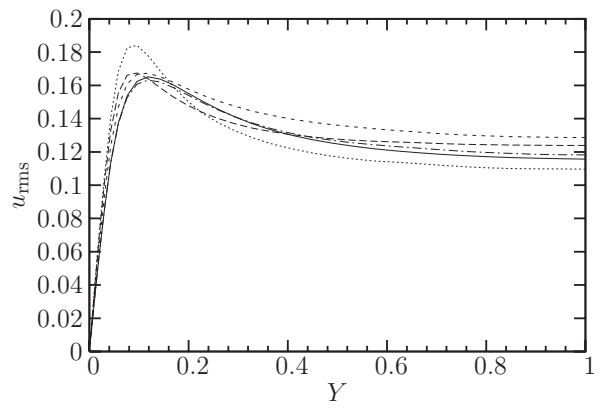

FIG. 6. Profiles of the rms values of fluctuations in the streamwise component of velocity (linestyles are given in Table I). For the reference run, the peak is at $y_{+} \simeq 14$. In the sparse grid runs it is shifted towards the wall, both in absolute and in wall units. A difference is also observable in the values of $u_{\mathrm{rms}}$, both at the peak and at the center of the channel.

\section{NEAR-WALL STRUCTURES AND SPECTRA}

The above results suggest that the main differences between the reference simulation and the sparse grid runs must reside in the near-wall structures where the high vertical gradients in the viscous layer need to be resolved correctly. Even though, in our nonisotropic implementation of the sparse-grid scheme, no mode reduction has been applied on the wall-normal Chebyshev decomposition, it is reasonable to assume that the typical length scales at a certain distance from the wall are of the same order of magnitude in all directions, thus explaining how the mode reduction on wallparallel directions can affect in particular the dynamics of this region. However, the modulus of the highest represented mode is the same in the sparse grid simulation as for the one at full resolution. What varies is the density with which each shell is represented.

As is well known, the regeneration mechanism of wall turbulence involves elongated streamwise vortices, which produce alternating streaks of fluid faster and slower than the average flow, the so-called high-speed and low-speed streaks [19-22]. These near wall structures are known from numerical and experimental data to have a characteristic spanwise spacing of around 100 wall units. This means that the dynamics selects a length scale $\lambda_{s} \sim 100 h / \mathrm{Re}_{*}$ in the spanwise direction. If the thinning-out of the modes takes place around this scale the dynamics of turbulence regeneration can be dramatically affected. Given the parameters of our simulations, the length scale of streamwise streaks is associated with a wave number $k_{s}=2 \pi / \lambda_{s} \sim 7.5$. For $n_{c}=8$, which is the least resolved case we consider, and for $L_{z}=2 \pi$, the streak wave number $k_{s}$ is on the border of the core group, and it is not surprising that streaks and streamwise vortices may be strongly influenced by a change in the rate of energy transfer at the border between two contiguous shells.

In Fig. 7, a typical snapshot of the streamwise component of velocity is shown, on a plane parallel to the wall, at $y^{+}$ $=2.2$, for each of the sparse-grid runs, and for the reference simulation. While the expected high- and low-speed streaks are present in all snapshots, their shape is qualitatively different at the lower resolutions. To characterize this quantitatively, the spanwise correlation of the downstream compo- 


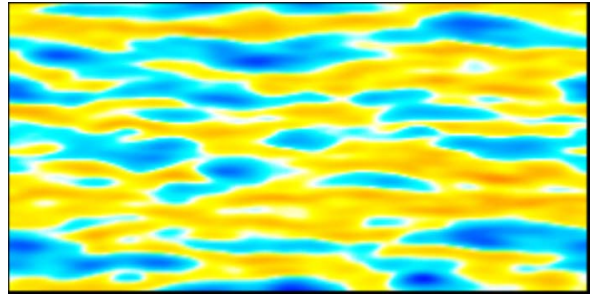

(a)

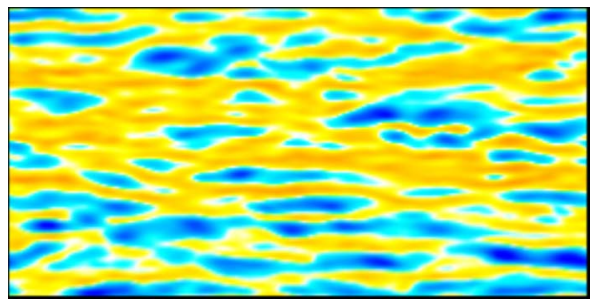

(b)



(c)

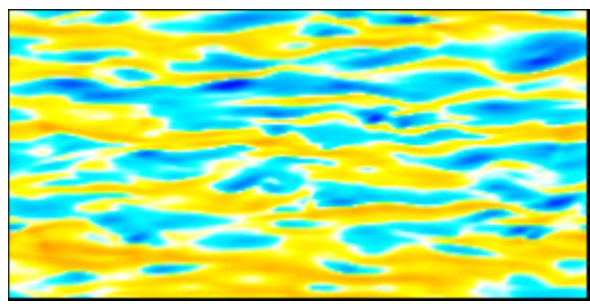

(d)

FIG. 7. (Color online) Streamwise component of velocity, at a distance $Y=0.03$ from the (upper) wall, for $n_{c}=8$ (a), $n_{c}=16$ (b), $n_{c}=24$ (c), and full resolution (d). Neglecting the minimal differences in wall shear stress, the distance corresponds to $y^{+}=3.6$, well within the viscous boundary layer. The average velocity has been subtracted. The yellow regions are faster, the blue regions slower than the mean velocity. White encodes the average velocity on the plane. The same color coding is used for all plots. In a grey scale, the light grey areas are fast, the darker ones slower. The elongated structures are low- and high-speed streaks, produced by fast fluid moving away from the wall and slow fluid coming in from the bulk, as a result of the longitudinal (hairpin) vortices which dominate the wall region. The visual differences between the flows are small, though one notes a broadening of structures as the resolution is reduced.

nent $u$ is shown in Fig. 8. This quantity is expected to go to zero and have a negative minimum at approximately half the typical distance between adjacent low speed streaks. The minimum falls between $y_{+}=50$ and $y_{+}=60$ for the full simulations, in substantial agreement with what can be expected. While for the larger $n_{c}$ 's this position is not very different,

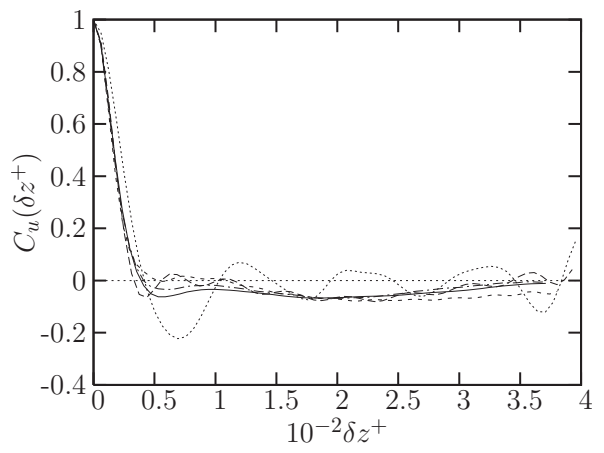

FIG. 8. Correlation of the downstream component of the velocity in the spanwise direction, at $y+=3.5$. The position of the first minimum in the correlation can be taken as half the typical separation of streaks in the near wall region. While at $n_{c}=8$ the correlation presents larger oscillations and a strongly shifted minimum, the variations become weaker for higher resolution, and very good agreement with the full simulation is reached for $n_{c} \geqslant 24$ (see Table I for linestyles).

for $n_{c}=8$ the minimum is deeper and the position shifted towards larger separation.

The differences between the various resolutions cannot be due to a lack of higher wave number modes, which are present in the model, but rather due to the fact that the higher modes are not active, or less active than in the full resolution case. Indeed, this is supported by the power spectra of the velocity field. The spectra in Fig. 9 show that, for each value of $n_{c}$, the energy content of the modes drops off sharply at the corresponding wave number. This drop is preceeded by a slight pile-up on larger wave numbers.

Actually, in the scheme we adopted, $n_{c}$ correspondes to different wave numbers in the downstream and the spanwise directions. However, since the spanwise size $L_{y}$ is half of the length of our channel $k_{x}^{b}<k_{y}^{b}$, the latter gives the smallest scale resolved in the core shell. This drop in energy was already observed in Ref. [7] in isotropic MHD turbulence. This pile-up/drop pattern is connected with a bottleneck in energy transfer, and can be explained by the sudden change in the density of modes at the transition between nearby shells $[33,34]$.

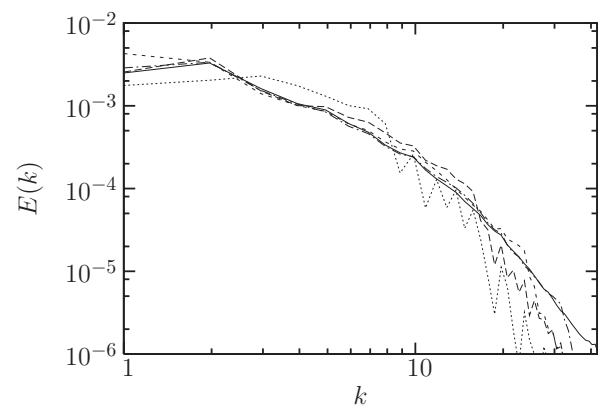

FIG. 9. Power spectra of velocity at the central horizontal plane (see Table I for linestyles). The kinks in the spectra are found to be in correspondence with the largest spanwise wave numbers in the core shell. The noisyness of the spectra after such kinks is due to the coarsening of the Fourier grid: since the spectra are computed on the original grid, the presence of many null modes produces the observed oscillations. 

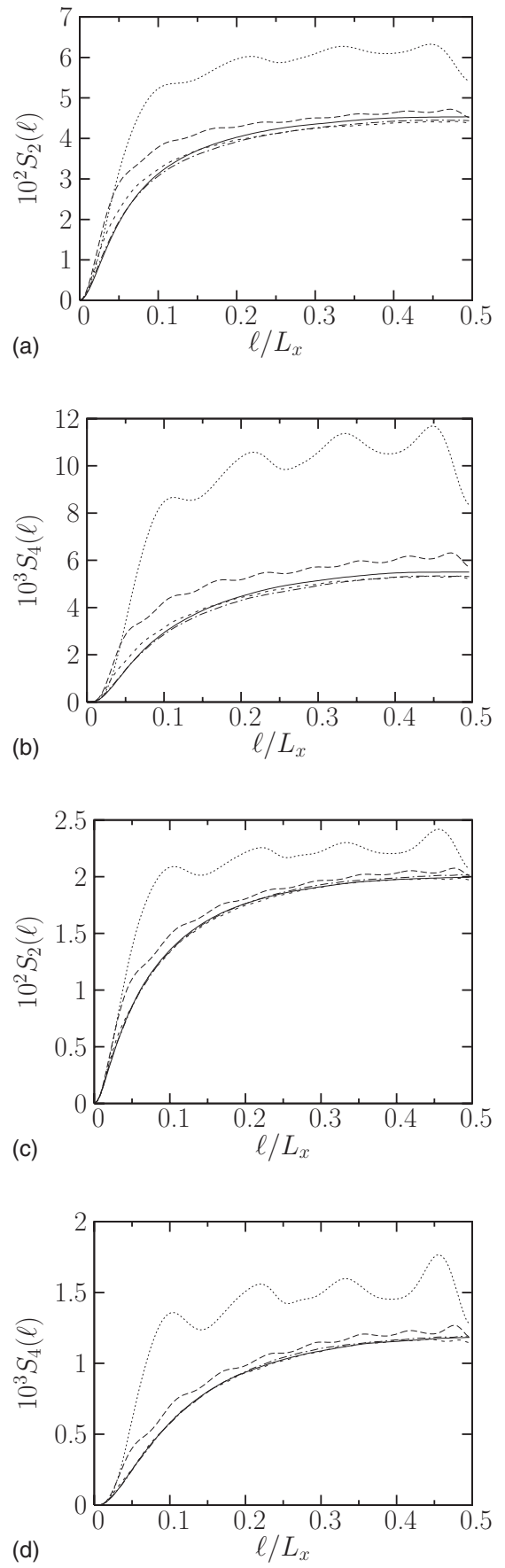

FIG. 10. Second and fourth order streamwise longitudinal structure functions of velocity, at $Y=0.12$, (a) and (b), and in the center plane $Y=h=1$, (c) and (d). For all plots it is evident that the lowest resolution $n_{c}=8$ is insufficient to reproduce the correct statistics. The results for $n_{c}=16$ are better, but only the higher resolutions agree with the results at full resolution within statistical uncertainty (see Table I for linestyles).

\section{STRUCTURE FUNCTIONS}

To further test the flow statistics in our simulations, we computed structure functions of the velocity field. In particular, we focus on the longitudinal structure functions of the streamwise component
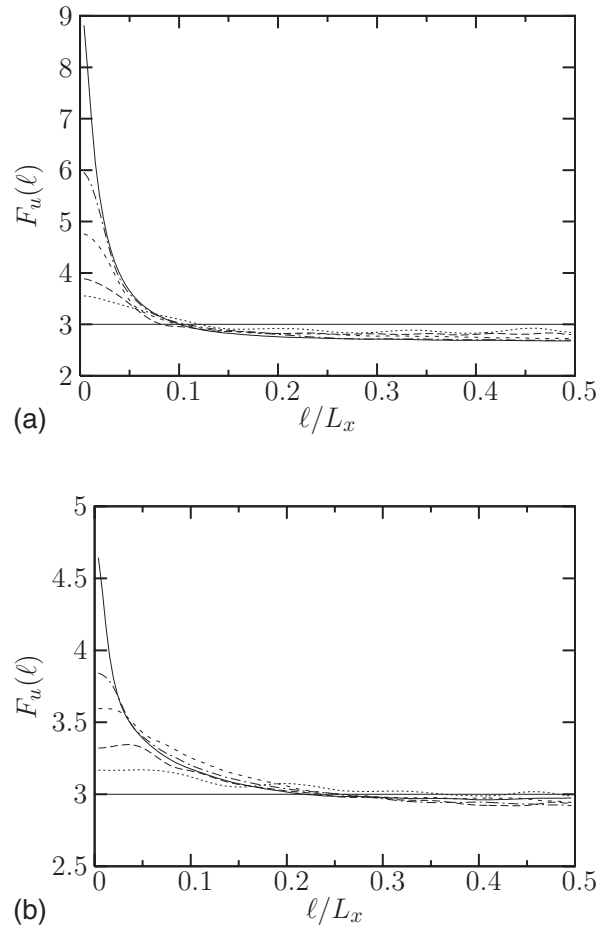

FIG. 11. Flatness of the downstream fluctuations of $u$, for $Y$ $=0.12$ (a) and $Y=h=1$ (b) (the same planes as in Fig. 10). While all simulations give values close to the Gaussian (which is 3 indicated by the horizontal full line) at large separations, small scale fluctuations exhibit a lower flatness for sparse grid simulations than for the reference one (see Table I for linestyles).

$$
S_{n}(l, y)=\left\langle\left[u\left(\mathbf{x}+l \mathbf{e}_{x}\right)-u(\mathbf{x})\right]^{n}\right\rangle_{y} .
$$

While the numerical values vary for different planes, the qualitative conclusions are the same for all plots shown in Fig. 10. As noted for the other observables, the run at $n_{c}=8$ shows the strongest differences. The shortcomings of the $n_{c}$ $=16$ simulation at small scales found in the the spanwise correlation are also reflected in the structure functions. With increasing $n_{c}$ the agreement with the results at full resolution improves. From the comparison between structure functions of fixed order at different distances from the wall, it is clear that the quality of results is independent of $Y$, as could also be induced from the spectra. The observation here is that the main drawback of the shell structure of the grid we used is to deplete the energy transfer through the scales. Since the energy spectrum of a function is the Fourier transform of its correlator (according to the Wiener-Kinchin formula), it is not surprising that $S_{2}(l)=2\left[\left\langle u^{2}\right\rangle-C(l)\right]$ bears a trace of the same effect $[33,34]$.

Due to the differences in the second moments for the different resolutions, the values of the fourth moment cannot be directly compared. We therefore study the flatness $F_{u}(l)$ $=S_{4}(l) /\left[S_{2}(l)\right]^{2}$ of the probability density function $P\left(\delta_{l} u\right)$ of velocity differences. First of all we emphasize that, while for homogeneous turbulence the asymptotic value of $\left\langle u^{4}\right\rangle /\left\langle u^{2}\right\rangle^{2}$ is expected to be 3, i.e., as for Gaussian random fields, in wall bounded turbulence it is known to be different, and to vary (nonmonotonically) with the distance from the wall. 
However, outside the viscous sublayer, it is generally very close to 3 . Figure 11 shows that at large scales the reduced simulations give results compatible with the full one (which deviates slighlty from the Gaussian value). On small scales there is a decrease in the flatness as the number of modes is reduced. This kind of trend was already noted in Ref. [12], together with a reduced intermittency.

\section{DISCUSSION AND CONCLUSIONS}

We have presented numerical tests of an approach to the simulation of turbulent boundary layers. In the normal direction, full resolution is kept. The method exploits a Fourier spectral decomposition of the velocity fields in the spanwise and streamwise directions. It fully resolves the large scales and reduces the number of modes used to describe the small scales. Modes on all scales are dynamically active, but the reciprocal Fourier grid is progressively reduced. In contrast to other reduction methods such as LES, no a priori modeling of the turbulent dynamics is introduced: the structure of the momentum equations is preserved, but interactions outside the active grid are pruned. By keeping the full resolution in normal direction, the near-wall resolution typical of the Gauss-Lobatto grid on which the Chebyshev decomposition is performed, is preserved.
The deficiencies of such a spectral reduction approach were already pointed out in previous works, and are also present in this implementation: in particular, bottlenecks in the down-scale spectral energy transfer and a decrease in the intermittency of the velocity fields are observed. In addition, we measured an increase in the momentum flux, which, in our geometry, is everywhere equal to the wall stress $J \equiv \tau$.

An unexpected result of our simulations is that they reproduce a logarithmic profile, and that its slope is in close agreement with the von Karman constant found in shear flows. The larger shear stress is reflected in lower values of the additive constant in the logarithmic law of the wall.

The results presented here are encouraging and show that the sparse grid approach to the simulation of boundary layers can give efficient and accurate results. Simulations at higher resolution and for larger Re are within reach and will further improve our understanding of the effects of the sparse grid on the properties of the simulated flows. They will also shed light on the question as to how and in which quantities small scale fluctuations affect boundary layers.

\section{ACKNOWLEDGMENTS}

We acknowledge the contribution of J. Bührle to the code we used for full simulations. This work was supported by the Deutsche Forschungsgemeinschaft.
[1] P. Moin and K. Mahesh, Annu. Rev. Fluid Mech. 30, 539 (1998).

[2] S. B. Pope, New J. Phys. 6, 35 (2004).

[3] R. S. Rogallo and P. Moin, Annu. Rev. Fluid Mech. 16, 99 (1984).

[4] M. Lesieur and O. Metais, Annu. Rev. Fluid Mech. 28, 45 (1996).

[5] C. Meneveau and J. Katz, Annu. Rev. Fluid Mech. 32, 1 (2000).

[6] U. Piomelli and E. Balaras, Annu. Rev. Fluid Mech. 34, 349 (2002).

[7] M. Meneguzzi, H. Politano, A. Pouquet, and M. Zolver, J. Comput. Phys. 132, 32 (1996).

[8] J. Eggers and S. Grossmann, Phys. Fluids A 3, 1958 (1991).

[9] S. Grossmann and D. Lohse, Z. Phys. B: Condens. Matter 89, 11 (1992).

[10] S. Grossmann and D. Lohse, Phys. Fluids 6, 611 (1994).

[11] S. Grossmann and D. Lohse, Phys. Rev. E 50, 2784 (1994).

[12] S. Grossmann, D. Lohse, and A. Reeh, Phys. Rev. Lett. 77, 5369 (1996).

[13] S. Grossmann and D. Lohse, Phys. Rev. Lett. 67, 445 (1991).

[14] S. Grossmann and D. Lohse, Phys. Rev. A 46, 903 (1992).

[15] T. Becker and B. Eckhardt, Z. Phys. B: Condens. Matter 101, 461 (1996).

[16] U. Frisch, Turbulence (Cambridge University Press, Cambridge, 1995).

[17] C. Canuto, M. Y. Hussaini, A. Quarteroni, and T. A. Zang, Spectral Methods in Fluid Dynamics (Springer, New York, 1988).

[18] J. Kim, P. Moin, and R. Moser, J. Fluid Mech. 177, 133
(1987)

[19] R. L. Panton, Prog. Aerosp. Sci. 37, 341 (2001).

[20] F. Waleffe, Stud. Appl. Math. 95, 319 (1995).

[21] B. Hof, C. W. H. van Doorne, J. Westerwheel, F. T. M. Nieuwstadt, H. Faisst, B. Eckhardt, H. Wedin, R. R. Kerswell, and F. Waleffe, Science 305, 1594 (2004).

[22] T. M. Schneider, B. Eckhardt and J. Vollmer, Phys. Rev. E, 75, 066313 (2007).

[23] P. J. Schmid and D. S. Henningson, Stability and Transition in Shear Flows (Springer, Berlin, 2001).

[24] O. Kitoh, K. Nakabyashi, and F. Nishimura, J. Fluid Mech. 539, 199 (2005).

[25] K. H. Bech, N. Tillmark, P. H. Alfredsson, and H. I. Andersson, J. Fluid Mech. 286, 291 (1995).

[26] S. B. Pope, Turbulent Flows (Cambridge University Press, Cambridge, 2000).

[27] P. A. Davidson, Turbulence (Oxford University Press, Oxford, 2004).

[28] L. D. Landau and E. M. Lifshitz, Fluid Mechanics (Pergamon Press, London, 1995).

[29] M. Oberlack, J. Fluid Mech. 427, 299 (2001).

[30] B. Lindgren, J. M. Österlund, and A. V. Johansson, J. Fluid Mech. 502, 127 (2004).

[31] N. Marati, J. Davoudi, C. M. Casciola, and B. Eckhardt, J. Turbul. 7, 1 (2006).

[32] J. Komminaho, A. Lundbadh, and A. Johansson, J. Fluid Mech. 320, 259 (1996).

[33] D. Lohse and A. Müller-Groeling, Phys. Rev. Lett. 74, 1747 (1995).

[34] D. Lohse and A. Müller-Groeling, Phys. Rev. E 54, 395 (1996). 